Research Paper

\title{
The feasibility of emergency medical technicians performing intermittent high-quality cardiopulmonary resuscitation
}

\author{
Chun-Hao Chang, ${ }^{1}$ Yi-Ju Hsu' ${ }^{1}$, Fang Li1, Yuan-Shuo Chan², Ching-Ping Lo ${ }^{3,4}$, Guan-Jian Peng, \\ Chin-Shan $\mathrm{Ho}^{1 \bowtie}$, Chi-Chang Huang ${ }^{1 凶}$ \\ 1. Graduate Institute of Sports Science, National Taiwan Sport University, Taoyuan, Taiwan. \\ 2. Department of Special Education, National Taipei University of Education, Taipei, Taiwan. \\ 3. College of Exercise and Health Science, National Taiwan Sport University, Taoyuan, Taiwan \\ 4. Ching Shuei Emergency Medical Service Team Of 5th Corps, Fire Department, New Taipei City Government, New Taipei City, Taiwan. \\ 5. Second Special Search and Rescue Branch, Special Search and Rescue Corps, Fire Department, Taoyuan City Government, Taoyuan City, Taiwan.
}

$\triangle$ Corresponding authors: Equal contributions of co-corresponding authors. Chin-Shan Ho, Ph.D. Graduate Institute of Sports Science, National Taiwan Sport University, Taoyuan 333325, Taiwan. Tel: +886-3-3283201 ext: 2425, E-mail: kilmur33@gmail.com; Chi-Chang Huang, Ph.D. Graduate Institute of Sports Science, National Taiwan Sport University, Taoyuan 333325, Taiwan. Tel: +886-3-3283201 ext: 2409, E-mail: d301090007@gmail.com.

(C) The author(s). This is an open access article distributed under the terms of the Creative Commons Attribution License (https://creativecommons.org/licenses/by/4.0/). See http://ivyspring.com/terms for full terms and conditions.

Received: 2021.02.24; Accepted: 2021.04.15; Published: 2021.04.29

\begin{abstract}
Background: Whether intermittent chest compressions have an effect on the quality of CPR is worthy of discussion. The purpose of this study was to investigate differences in the chest compression quality of emergency medical technicians (EMTs) performing cardiopulmonary resuscitation (CPR) with different rest intervals.

Methods: Seventy male firefighters with EMT licenses participated in this study. Participants completed body composition measurements and three CPR quality tests, as follows: (1) CPR-uninterrupted for 10 minutes; (2) after 2 days of rest, CPR 10s-intermittent (CPR-10s), for 2 minutes each time and 5 cycles; (3) after another 2 days of rest, CPR 20s-intermittent (CPR-20s), for 2 minutes each time and 5 cycles.

Results: Body composition results showed that body mass (BM), body mass index (BMI), upper limb muscle mass (ULMM), core muscle mass (CMM), and upper limb-core muscle mass (UL+CMM) were positively correlated with chest compression depth $(C C D)(p<0.05)$. Analysis of the three different modes of CPR quality analysis indicated significant differences in the chest compression fraction $(C C F, F=6.801, p=0.001)$, chest compression rebound rate $(C C R R, F=3.919, p=0.021)$, and ratings of perceived exertion $(\mathrm{RPE}, \mathrm{F}=$ $23.815, p<0.001)$. Among the different performance cycles of CPR-10s, significant differences were found in CCF, CCD, CCR (chest compression rate), and RPE $(p<0.05)$. On the other hand, among the different performance cycles of CPR-20s, significant differences were found in CCD, CCR, and RPE $(p<0.05)$. Moreover, the CCF, CCD, and RPE scores of the two tests reached significant differences in specific phases $(p$ $<0.05$ ).

Conclusions: This study confirmed that the upper limb muscle mass or the weight of the upper body of EMTs is positively correlated with the quality of CPR. In addition, intermittent chest compressions with safe interruption intervals can reduce fatigue caused by long-term chest compressions and maintain better chest compression quality.
\end{abstract}

Key words: CPR; out-of-hospital cardiac arrest; chest compression fraction; fatigue; emergency medical technician

\section{Introduction}

Out-of-hospital cardiac arrest (OHCA) is the destructive manifestation of coronary artery disease and commonly leads to high morbidity and mortality [1]. Nevertheless, it is recognized that high-quality cardiopulmonary resuscitation (CPR) is a vital determinant of survival following cardiac arrest [2-6] and may achieve the return of spontaneous circulation (ROSC) by providing the required blood flow to preserve significant organ functions [1, 7-11]. According to the American Heart Association (AHA) 
Guidelines for CPR and Emergency Cardiovascular Care, high-quality CPR involves an adequate compression rate and depth, minimized interruptions in chest compressions, full chest rebound, and avoidance of excessive ventilation [12]. Of these, effective chest compressions are the most critical aspect of high-quality CPR [2, 11, 13]. For manual $\mathrm{CPR}$, the AHA recommends an adult compression depth of at least $5 \mathrm{~cm}$ but no greater than $6 \mathrm{~cm}$ and a chest compression rate of 100 to 120 beats/min [12, 14].

Adequate chest compressions during CPR are the basis for providing ample blood flow to protect vital organs. However, the quality of manual chest compressions is related to the body weight, body composition, and physical fitness of the rescuer. In a previous study, the relationship between the body weight of rescuers and chest compression quality during CPR was pointed out. The values of oxygen uptake and ratings of perceived exertion (RPE) were dramatically higher in the light group than in the heavy group. In contrast, the heavy group utilized their body weight as a compression force during chest compressions without becoming rapidly fatigued and generally could maintain the ratio of adequate chest compressions $[10,15,16]$. Similarly, there is a positive correlation between high BMI and high-quality CPR. Rescuers with higher BMI showed better chest compression performance and less fatigue, while rescuers with relatively lower BMI showed lower chest compression performance [17]. Furthermore, according to Kaminska et al. (2018), fat-free mass, trunk muscle mass, left or right arm muscle mass and basal metabolic rate are positively correlated with the depth of manual chest compressions [18].

In clinical settings, rescuers usually have to perform CPR in a kneeling or standing posture with their hands on the patient's sternum while keeping the upper limbs upright $[19,20]$, which is a very physically exhausting posture and movement. It can be found that a progressive reduction in the percentage of correct chest compressions after 1 minute of chest compressions [15, 21, 22]. The reason for the decreasing trend in correct chest compressions could be explained by the rescuer's fatigue, which causes a dramatic decline in the quality of CPR [23]. According to statistics from the National Fire Agency of the Ministry of the Interior of Taiwan, the emergency care time (pre-hospital time) after EMTs come into contact with a patient totals 16.64 minutes, and only one EMT cares for the patient in the back of the vehicle on the way to the hospital. Thus, the attending personnel must make a judgment between the quality and time of the first aid. In the case of patients with OHCA, the EMT needs to continue CPR without interruption [24]. Therefore, maintaining the quality of CPR is crucial in the long-term pre-hospital stage.

Few studies have evaluated changes in the quality of CPR when rescuers are allowed to rest intermittently during continuous chest compressions. In the study of Min, et al. (2013), the subjects applied three different continuous chest compression methods on a manikin model for $10 \mathrm{~min}$, including continuous chest compressions without a break (CCC), a 10-s break after 100 chest compressions (10/100), and a 10 -s break after 200 chest compressions (10/200). The outcomes indicated that the 10/100 method had the highest ratio of adequate chest compressions, followed by the 10/200 and CCC methods, which could be attributed to the rest periods [25]. If rescuers are provided with rest breaks during continuous chest compressions, their fatigue will be reduced and the quality of CPR will be improved. The length of the interruptions will affect the survival rate [26], and according to the AHA (2015) Guidelines for CPR and Emergency Cardiovascular Care, the chest compressions should be interrupted as little as possible to maintain the survival rate [12]. However, when the manpower is insufficient or the transportation is difficult, there may be no way to perform chest compressions without interruption and thereby maintain the quality of CPR until arrival at the hospital. Perhaps, under the premise of proper care and life monitoring, temporary interruption of chest compressions for rest should be a feasible method.

In view of the above, we assume that resting for a limited interruption time can maintain the CPR compression quality and reduce the fatigue caused by long-term chest compressions. It has also been proposed by previous researchers that the interruption time of chest compression should not exceed 20 seconds [14, 27-29]. As far as our knowledge is concerned, there should be no research to compare the effects of different chest compression interruption time on the quality of CPR. Therefore, the purposes of the present study were first, to investigate the correlation between the quality of $\mathrm{CPR}$ and the body composition of the emergency medical technician (EMT), and second, to evaluate the impacts of different chest compression methods on the quality of CPR.

\section{Method and Methods}

\section{Study design}

This study recruited firefighters who had received relevant professional training so as to examine the correlation between the quality of $\mathrm{CPR}$ 
performance and body composition and to understand the impacts of different methods of chest compression, two with interruption times, on the quality of CPR. Participants performed CPR quality tests of three chest compression methods on the Laerdal ${ }^{\circledR}$ Little Anne QCPR (Laerdal Medical, Stavanger, Norway) manikin. The study procedure was approved by the Institutional Review Board of $\mathrm{Fu}$ Jen Catholic University (New Taipei City, Taiwan) (reference number: C108165). Before the experimental tests began, all subjects completed informed consent forms.

\section{Participants}

A total of 70 active male firefighters from different fire departments in northern Taiwan volunteered to participate in the study. In Taiwan, firefighters are responsible for firefighting and ambulance services, so the recruitment of participants is mainly firefighters. All participants needed to have Emergency Medical Technician-Intermediate (EMT-2) licenses or higher, as well as experience with actual emergency rescue work and CPR first aid. EMT-2 must complete a 24-hour re-training course each year, and Emergency Medical Technician-Paramedic (EMT-P) must complete a 32-hour re-training course each year, both of which include CPR training. The exclusion criteria were diagnoses of cardiovascular disease, chronic disease, or unsuitability for intense exercise. The anthropometry and body composition characteristics of the participants are listed in Table 1.

Table 1. Anthropometry and body composition characteristics of participants

\begin{tabular}{lll}
\hline & Mean \pm SD & Range \\
\hline Age $($ years $)$ & $30.00 \pm 5.04$ & $21.0-39.0$ \\
Height $(\mathrm{cm})$ & $175.49 \pm 5.03$ & $165.0-186.0$ \\
Body mass $(\mathrm{kg})$ & $77.79 \pm 12.38$ & $54.3-117.9$ \\
BMI $\left(\mathrm{kg} / \mathrm{m}^{2}\right)$ & $25.22 \pm 3.61$ & $18.5-35.2$ \\
Percentage of body fat $(\%)$ & $20.21 \pm 6.03$ & $9.9-35.3$ \\
Basal metabolic rate $(\mathrm{kcal} /$ day) & $1699.17 \pm 153.10$ & $1378-2057$ \\
Skeletal muscle mass $(\mathrm{kg})$ & $34.97 \pm 4.28$ & $25.7-45.0$ \\
Upper limb muscle mass $(\mathrm{kg})$ & $6.98 \pm 1.07$ & $4.5-9.4$ \\
Core muscle mass $(\mathrm{kg})$ & $27.34 \pm 3.15$ & $19.7-34.2$ \\
Upper limb+Core muscle mass $(\mathrm{kg})$ & $34.32 \pm 4.22$ & $24.23-43.6$
\end{tabular}

Mean \pm SD, mean value \pm standard deviation. BMI, body mass index.

\section{Body composition measurement}

Body composition was evaluated by standard procedures [30]. Participants' body composition parameters were measured with the InBody ${ }^{\circledR} 570$ Body Composition Analyzer (Biospace, Inc. Seoul, Korea), for which their ages and heights were entered. Height was measured with an H900 heightmeasurement scale (NAGATA Scale Co., Ltd. Tainan, Taiwan). The InBody ${ }^{\circledR} 570$ is a reliable method of estimating body composition, including skeletal muscle mass, percentage of body fat, and resting metabolic rate (RMR), with a multi-frequency bioelectrical impedance analyser [30]. During the body composition measurement, all participants wore lightweight sportswear and removed their shoes, socks, and any metal objects.

\section{CPR quality measurement}

All participants completed three CPR quality tests in random order. They were required to rest for at least 2 days between each test, and to prevent muscle fatigue or feelings of tiredness, no additional exercise training or weight-bearing work was allowed during the rest period. Before the test, it is necessary to confirm whether the participant has taken 2 days of rest, including at least one day's holiday, and ask whether the participant feels unwell or fatigued before proceeding to the next stage of the test. For all three tests, the participants followed the chest compression technical specifications stipulated by the 2015 AHA guidelines (compression depth of 50-60 $\mathrm{mm}$, compression rate of 100-120 times/min, no interruptions, and full rebound of the chest) [12], with the only differences being the exclusion or inclusion, and the lengths, of the interruptions. No feedback device was used during the tests.

For the CPR-uninterrupted test, the participants performed chest compressions for 10 minutes, without interruptions. Immediately after the test, participants rated their current degree of fatigue degree on the Borg Rating of Perceived Exertion (RPE) scale.

For the CPR 10s-intermittent (CPR-10s) test, the participants performed 5 sets of continuous chest compressions, each lasting 2 minutes and separated by 10 seconds of rest. Immediately after the test, participants rated their current degree of fatigue degree on the Borg RPE scale.

For the CPR 20s-intermittent (CPR-20s) test, the participants performed 5 sets of continuous chest compressions, each lasting 2 minutes and separated by 20 seconds of rest. Immediately after the test, participants rated their current degree of fatigue degree on the Borg RPE scale.

\section{Data Analysis}

All 70 participants completed the study tests. The data from the InBody ${ }^{\circledR} 570$ Body Composition Analyzer and Laerdal ${ }^{\circledR}$ Little Anne QCPR Manikin, as well as the ratings of perceived exertion (RPE), were all exported to Microsoft Excel (Microsoft Office 2013 for Windows). The body composition parameters of body mass, body mass index, skeletal muscle mass, percentage of body fat, upper limb muscle mass, core 
muscle mass, upper limb-core muscle mass, and basal metabolic rate of the InBody ${ }^{\circledR} 570$ Body Composition Analyzer were used in this study. The data of the Laerdal ${ }^{\circledR}$ Little Anne QCPR Manikin were calculated in the SimPad PLUS device (Laerdal, Stavanger, Norway) with activated SkillReporter software. The chest compression quality parameters of chest compression fraction (CCF), chest compression depth (CCD), chest compression rate (CCR), and chest compression rebound rate (CCRR) were used in this study.

\section{Statistical Analysis}

All data are presented as means \pm standard deviation (SD). Pearson's coefficient of determination was used to analyze the correlation between body composition and CPR-uninterrupted $\mathrm{CPR}$ quality data. One-way analysis of variance (ANOVA) was used to compare the CPR quality of the different chest compression operations. One-way repeated measures ANOVA was used to investigate differences in CPR quality at each phase of the two intermittent times (CPR-10s and CPR-20s tests), respectively. The paired sample t-test was used to analyze the difference in the quality parameters of chest compressions in each phase of CPR-10s and CPR-20s tests. All statistical analyses were performed in IBM SPSS Statistics version 20 (IBM Corp., New York, NY, USA). The significance level was set to $\alpha<0.05$.

\section{Results}

\section{Correlation between body composition and CPR quality}

The correlation results of body composition and CPR-uninterrupted CPR quality are provided in Table 2. It was found that BM, BMI, ULMM, CMM and $\mathrm{UL}+\mathrm{CMM}$ were positively correlated with CCD (BM: $\mathrm{r}=0.315, p=0.008$; BMI: $\mathrm{r}=0.325, p=0.006$; ULMM: $\mathrm{r}$ $=0.411, p<0.001 ; \mathrm{CMM}: \mathrm{r}=0.393, p=0.001$; UL+CMM: $r=0.398, p=0.001)$. There were no significant correlations between CPR quality and body fat percentage $(\mathrm{PBF})(p>0.05)$.

Table 2. Pearson correlation $(r)$ between body composition and CPR quality

\begin{tabular}{llllll}
\hline & CCF & CCD & CCR & CCRR & RPE \\
\hline BM & 0.144 & $0.315^{*}$ & 0.073 & -0.059 & -0.042 \\
PBF & -0.010 & 0.066 & 0.134 & 0.033 & 0.060 \\
BMI & 0.161 & $0.325^{*}$ & 0.076 & -0.009 & -0.109 \\
ULMM & 0.217 & $0.411^{*}$ & -0.016 & -0.102 & -0.143 \\
CMM & 0.199 & $0.393^{*}$ & -0.011 & -0.110 & -0.128 \\
UL+CMM & 0.204 & $0.398^{*}$ & -0.012 & -0.108 & -0.132 \\
\hline
\end{tabular}

${ }^{*} p<0.05$. BM, body mass. PBF, percentage of body fat. BMI, body mass index. ULMM, upper limb muscle mass. CMM, core muscle mass. UL+CMM, upper limb muscle mass plus core muscle mass. CCF, chest compression fraction. CCD, chest compression depth. CCR, chest compression rate. CCRR, chest compression rebound rate. RPE, rating of perceived exertion.

\section{CPR quality of different CC interruption times}

The ANOVA results for each CPR quality of the CPR-uninterrupted, CPR-10s, and CPR-20s tests are listed in Table 3. The CCF values CPR-uninterrupted $(92.70 \pm 9.49 \%)$ was significantly lower than CPR-20s $(97.08 \pm 3.32 \%)(\mathrm{F}=6.801, p=0.001)$. The CCRR values CPR-uninterrupted $(76.83 \pm 30.88 \%)$ was significantly lower than CPR-20s (87.41 $\pm 20.93 \%)(\mathrm{F}=3.919, p=$ 0.021).The RPE values CPR-uninterrupted (14.96 \pm 2.58) were significantly higher than those of CPR-10s $(13.00 \pm 2.12)$ and CPR-20s $(12.36 \pm 2.23)(\mathrm{F}=23.815, p$ $<0.001)$. However, No significant differences were found in CCD or CCR among the three chest compression methods $(p>0.05)$.

Table 3. Comparison of the CPR quality and fatigue with different chest compression interruption times

\begin{tabular}{lllll}
\hline & \multicolumn{2}{l}{ CPR-uninterrupted CPR-10s } & CPR-20s & $p$ \\
\hline CCF $(\%)$ & $92.70 \pm 9.49 \neq$ & $95.10 \pm 6.87$ & $97.08 \pm 3.32^{*}$ & 0.001 \\
CCD $(\mathrm{mm})$ & $56.27 \pm 5.03$ & $56.52 \pm 4.13$ & $57.41 \pm 3.65$ & 0.264 \\
CCR (beats/min) & $110.09 \pm 9.12$ & $109.01 \pm 7.44$ & $108.92 \pm 5.77$ & 0.601 \\
CCRR $(\%)$ & $76.83 \pm 30.88 \neq$ & $86.77 \pm 22.21$ & $87.41 \pm 20.93^{*}$ & 0.021 \\
RPE & $14.96 \pm 2.58 \dagger \neq$ & $13.00 \pm 2.12^{*}$ & $12.36 \pm 2.23^{*}$ & $<0.001$ \\
\hline
\end{tabular}

*Significantly different from CPR-uninterrupted, $p<0.05$. Significantly different from CPR-10s, $p<0.05$. $\ddagger$ Significantly different from CPR-20s, $p<0.05$. CCF, chest compression fraction. CCD, chest compression depth. CCR, chest compression rate. CCRR, chest compression rebound rate. RPE, rating of perceived exertion.

\section{Comparison of CPR quality between CPR-10s and CPR-20s tests}

The changes in the CPR quality over the 5 cycles of chest compressions of the CPR-10s and CPR-20s test are listed in Table 4 . For the various CPR quality parameters in the CPR-10s test, significant decreases were found in CCF $(96.13 \pm 6.04 \%$ to $92.97 \pm 12.74 \%, p$ $=0.042), \operatorname{CCD}(58.46 \pm 2.93 \mathrm{~mm}$ to $55.11 \pm 5.59 \mathrm{~mm}, p<$ $0.001)$, and then CCR $(107.96 \pm 6.92$ beats $/ \mathrm{min}$ to $110.93 \pm 7.74$ beats $/ \mathrm{min}, p<0.001)$ and RPE $(10.81 \pm$ 1.70 to $14.73 \pm 2.81, p<0.001)$ with increases in the number of cyclic chest compressions, and no significant difference was found in CCRR $(p=0.109)$. For the various CPR quality parameters in the CPR-20s test, significant decreases were found in CCD $(58.80 \pm 2.88 \mathrm{~mm}$ to $56.69 \pm 3.67 \mathrm{~mm}, p=0.001)$, and then CCR $(107.91 \pm 5.82$ beats/min to $110.96 \pm 7.05$ beats/min, $p<0.001)$ and RPE $(10.47 \pm 2.12$ to $14.06 \pm$ $2.81, p<0.001)$ with increases in the number of cyclic chest compressions, but no significant changes were found in CCF $(p=0.429)$ or CCRR $(p=0.455)$ with increases in the number of cyclic chest compressions.

\section{The difference in the quality of chest compression between CPR-10s and CPR-20s tests}

The difference in the quality of chest compressions between the CPR-10s and CPR-20s tests 

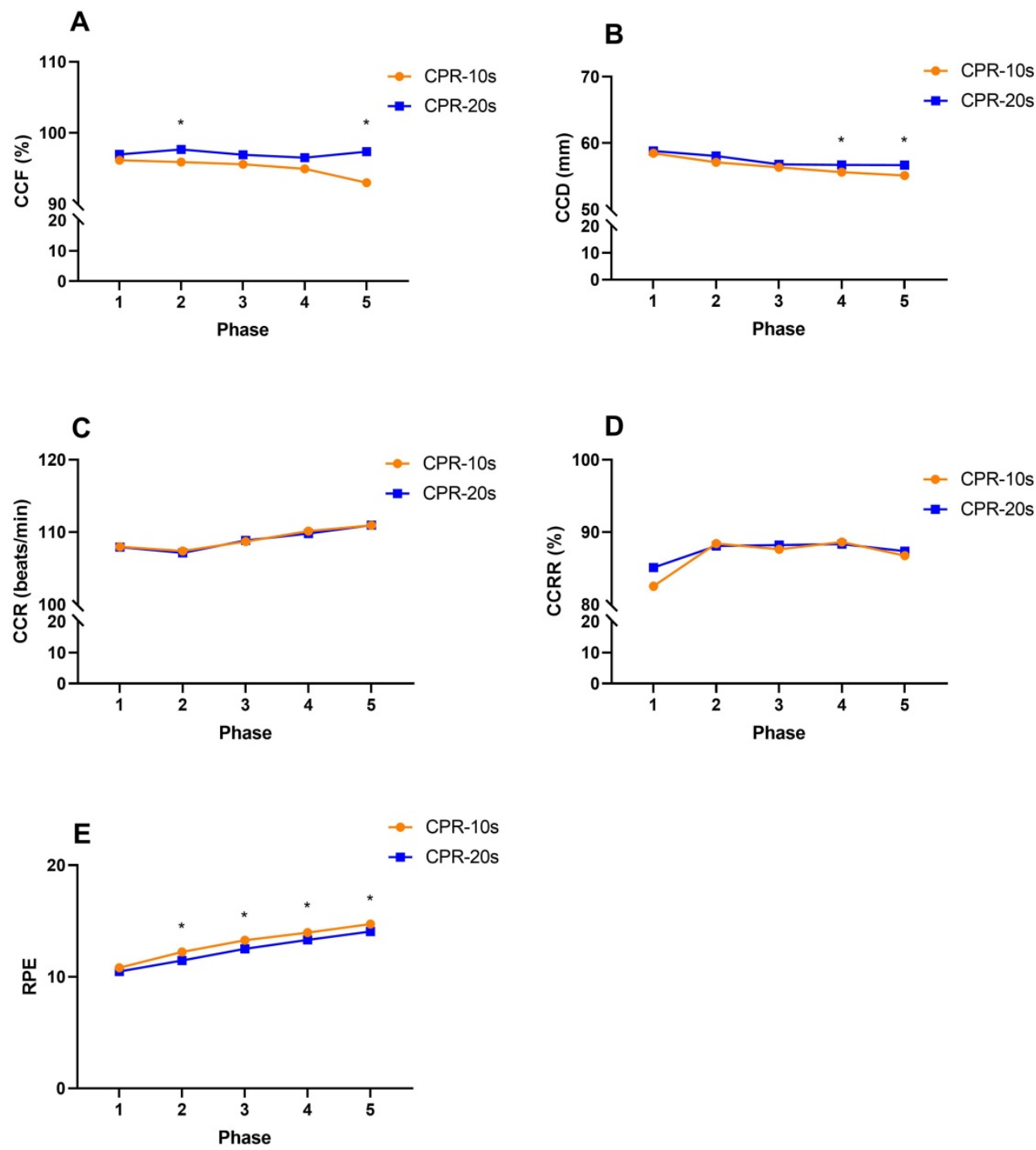

Figure 1. The difference in the quality of chest compression between CPR-10s and CPR-20s. A, CCF: chest compression fraction). B. CCD: chest compression depth. C, CCR: chest compression rate. D, CCRR: chest compression rebound rate. E, RPE: rating of perceived exertion. *Significantly different from CPR-10s, $p<0.05$.

in each phase is shown in Figure 1. The results showed that phase 2 (0.70 [95\% CI, -3.18 to -0.39$] ; p=$ $0.013)$ and phase 5 (1.35 [95\% CI, -7.06 to -1.68$] ; p=$ 0.013 ) of CCF reached a significant difference between the CPR-10s and CPR-20s tests; phase $4(0.50$ [95\% CI, -2.08 to -0.09$] ; \mathrm{p}=0.033)$ and phase $5(0.47$ [95\% CI, -2.51 to -0.62$] ; p=0.001$ ) of CCD reached a significant difference between the CPR-10s and CPR-20s tests; and phase 2 (0.22 [95\% CI, 0.34 to 1.21$] ; p=0.001)$, phase 3 (0.22 [ $95 \% \mathrm{CI}, 0.31$ to 1.20]; $p=0.001)$, phase 4 ( 0.23 [95\% CI, 0.18 to 1.11$] ; p=0.008)$ and phase $5(0.25$ [95\% CI, 0.18 to 1.17]; $p=0.009$ ) of RPE achieve significant difference between CPR-10s and CPR-20s test.

\section{Discussion}

High-quality chest compression is an important factor in determining patient survival. We investigated the relationship between the body composition of a firefighter and the quality of CPR, as well as the quality of CPR during chest compressions with different rest periods in this study. The results indicated that, in terms of upper limb muscle mass and core muscle mass, there was a significant positive correlation of body composition with CPR quality. This result is similar to the finding of Ogata, Fujimaru, \& Kondo (2015) that, in athletes or workers, physical training of upper body muscle strength is correlated with the quality of CPR or fatigue [31]. To reduce the deterioration of CPR quality in firefighters performing chest compressions without interruptions and to prevent hypoxia in the brains of OHCA patients due to excessive CPR interruption times, we assumed that a limited CPR interruption (rest) time could maintain the CPR quality of professional first responders while preventing premature muscle fatigue caused by prolonged chest compressions. We found that only CCRR had no significant difference in 
quality during the CPR-10s test. However, in the quality of CPR-20s, it can be found that CCF and CCRR did not change significantly with the increase of chest compression operation time. From these results, it appears that the CPR-20s intermittent method can maintain CPR quality for a long time.

The maintenance of high-quality CPR depends on the rescuer's technique and physical fitness. With increases in the first aid time, the rescuer's physical strength will gradually decrease, and this decrease will directly affect the quality of CPR. The results of body composition analysis showed that BM, BMI, ULMM, CMM, and UL+CMM were only positively correlated with $\mathrm{CCD}$ in the CPR quality index parameters. This shows that the above body composition parameters of the participants in this study only affect the depth of chest compressions, and do not affect other CPR quality index parameters. The reason may be that the participants already have proficient CPR skills and have good control in CPR operations. Only the body weight and other related parameters will affect the depth of chest compressions. Furthermore, the weight of their body or limbs can be used as the force during chest compression, which in turn affects the depth of compression. A study by Kaminska et al. (2018) pointed out that the basal metabolic rate, fat-free mass (FFM), trunk muscle mass, and left and right arm muscle mass of 100 medical students were positively correlated with chest compression depth. In addition, the chest compression depth can increase by $7.3 \mathrm{~mm}$ with every $1 \mathrm{~kg}$ increase in arm muscle mass [18]. These results strongly indicate that upper limb muscle mass or the weight of the upper body may be one of the factors affecting the quality of CPR.

When EMTs rapidly arrive on the scene for pre-hospital emergency care, the process of transport to the hospital can be viewed as a race of physical fitness against time. The study of Chonde et al. (2020) showed that the median time from arrival to departure of ambulance personnel was $20 \mathrm{~min}$ (interquartile range (IQR) 19-20 $\mathrm{min}$ ), and the time to the hospital was $34 \mathrm{~min}$ (IQR 30-45 $\mathrm{min}$ ), so the duration of pre-hospital CPR was 26 min (IQR 25-40 min) [32]. One of the main challenges in transporting OHCA patients to the hospital is the maintenance of high-quality CPR during the transport process, especially in difficult transport conditions [33]. Although past studies have found that interruption of chest compressions was a common cause of decreased overall CPR quality, and was associated with a poor prognosis [28, 34-36], it is likely that, if chest compressions can be performed during a long period of transportation or with a manpower shortage under rigorous monitoring of life monitoring equipment, it will be feasible to maintain the CPR quality of the EMT and reduce EMT fatigue through turn-taking, taking rests, or taking rescue breaths within short interruption times. The results of this study showed a trend of improvement in the CCF $(p=0.001)$ and CCRR $(p=0.021)$ scores of the three CPR tests (10-min uninterrupted, CPR-10s, and CPR-20s) with increases in the interruption time. However, in terms of degree of fatigue, the degree of fatigue after the 10-min uninterrupted test was significantly greater than those of the CPR-10s and CPR-20s tests $(p<0.001)$. When the skeletal muscles are activated continuously for a long time, fatigue is prone to appear early, and it will be difficult to maintain the target action. Through temporary rest, it is possible to postpone the early occurrence of fatigue. It should be noted that, because the time of chest compression interruption may affect the survival rate [26], the interruption time should be as short as possible $(<10-20$ s) to reduce the impact on neurological function, whether traditional or mechanical chest compression is used [14, 27-29]. In other words, the interruption time of chest compressions requires careful evaluation and monitoring to ensure the safety of OHCA patients.

Table 4. Changes in CPR quality and fatigue in different phases of the CPR-10s intermittent method

\begin{tabular}{|c|c|c|c|c|c|c|}
\hline & Phase 1 & Phase 2 & Phase 3 & Phase 4 & Phase 5 & $p$ \\
\hline \multicolumn{7}{|c|}{ CPR-10s intermittent method } \\
\hline CCF $(\%)$ & $96.13 \pm 6.04 \#$ & $95.89 \pm 5.77 \#$ & $95.57 \pm 7.89 \#$ & $94.94 \pm 8.59 \#$ & $92.97 \pm 12.74^{*} \dagger \neq \S$ & 0.042 \\
\hline $\mathrm{CCD}(\mathrm{mm})$ & $58.46 \pm 2.93 \dagger \neq \S \#$ & $57.11 \pm 4.37^{*} \neq \S \#$ & $56.34 \pm 4.76^{*} \dagger \S \#$ & $55.61 \pm 5.04^{*} \dagger \neq$ & $55.11 \pm 5.59^{*} \dagger \neq$ & $<0.001$ \\
\hline CCR (beats/min) & $107.96 \pm 6.92 \S \#$ & $107.37 \pm 8.09 \neq \S \#$ & $108.69 \pm 7.96 \dagger \S \#$ & $110.13 \pm 8.34^{*} \dagger \neq \#$ & $110.93 \pm 7.74^{*} \dagger \neq \S$ & $<0.001$ \\
\hline CCRR $(\%)$ & $82.50 \pm 28.64$ & $88.40 \pm 26.87$ & $87.60 \pm 25.30$ & $88.60 \pm 23.02$ & $86.74 \pm 24.60$ & 0.109 \\
\hline RPE & $10.81 \pm 1.70 \dagger \neq \S \#$ & $12.23 \pm 2.07^{*} \neq \S \#$ & $13.27 \pm 2.35^{*} \dagger \S \#$ & $13.96 \pm 2.56^{*} \dagger \neq \#$ & $14.73 \pm 2.81^{*} \dagger \neq \S$ & $<0.001$ \\
\hline \multicolumn{7}{|c|}{ CPR-20s intermittent method } \\
\hline CCF $(\%)$ & $96.96 \pm 4.20$ & $97.67 \pm 2.78$ & $96.91 \pm 5.98$ & $96.50 \pm 6.56$ & $97.34 \pm 3.39$ & 0.429 \\
\hline $\mathrm{CCD}(\mathrm{mm})$ & $58.80 \pm 2.88 \neq \S \#$ & $58.06 \pm 6.76$ & $56.80 \pm 4.37^{*}$ & $56.70 \pm 4.06^{*}$ & $56.69 \pm 3.67^{*}$ & 0.001 \\
\hline CCR (beats/min) & $107.91 \pm 5.82 \S \#$ & $107.11 \pm 6.01 \neq \S \#$ & $108.84 \pm 6.07 \dagger \S \#$ & $109.79 \pm 6.63^{*} \dagger \neq \#$ & $110.96 \pm 7.05^{*} \dagger \neq \S$ & $<0.001$ \\
\hline CCRR $(\%)$ & $85.09 \pm 26.71$ & $88.07 \pm 24.35$ & $88.20 \pm 22.22$ & $88.33 \pm 22.29$ & $87.34 \pm 22.15$ & 0.455 \\
\hline RPE & $10.47 \pm 2.12 \dagger \neq \S \#$ & $11.46 \pm 2.19^{*} \neq \S \#$ & $12.51 \pm 2.38^{*} \dagger \S \#$ & $13.31 \pm 2.45^{*} \dagger \neq \#$ & $14.06 \pm 2.81^{*} \dagger \neq \S$ & $<0.001$ \\
\hline
\end{tabular}

*Significantly different from Phase $1, p<0.05$. † Significantly different from Phase $2, p<0.05$. $\ddagger$ Significantly different from Phase $3, p<0.05$. $\S$ Significantly different from Phase 4, $p<0.05$. \# Significantly different from Phase 5, $p<0.05$. 
The 2015 AHA Guidelines Update for CPR and ECC stipulate the importance and operating specifications of high-quality CPR (CPR administration as early as possible, compression rate of $100-120 / \mathrm{min}, \mathrm{CCD}$ for adults of $50-60 \mathrm{~mm}, \mathrm{CCD}$ for infants and children of at least $1 / 3$ of the thickness of the chest, full rebound of the chest, and minimized time and frequency of chest compression interruptions) [12]. The results of this study showed that, during increases in the total chest compression time during the CPR-10s test, there were significant decreases in CPR quality in the CPR quality index parameters of CCF, CCD, CCR, and RPE $(p<0.05)$, and only CCRR remained unchanged $(p>0.05)$. In contrast, the results of the CPR-20s test showed that only significant decreases in CPR quality in CCD, CCR and RPE $(p<0.05)$ with increases in chest compression time, while CCF and CCRR did not change significantly $(p>0.05)$. Moreover, the CCF, CCD, and RPE scores of the two tests reached significant differences in specific phases. The CPR-20s method appears to be better able than the CPR-10s method for maintaining CPR quality for extended periods. It can be seen that whether chest compressions are interrupted for rest and the length of the rest period will affect the quality of chest compressions and the degree of fatigue. This finding is similar to that of Zou et al. (2015) [37]. When the chest compression rate increases, the chest rebound and compression depth scores worsen, and the onset of fatigue occurs significantly earlier. In the results of this study, only CCRR was not affected by increases in chest compression time.

Many factors affect whether an OHCA patient can achieve ROSC. If each stage can be linked together, the chance of rescuing a person will be greatly improved. It is not difficult to understand that whether OHCA patients receive chest compressions is the focus of the entire CPR process. Since the emergency treatment time (pre-hospital time) after the EMT comes into contact with the patient may be affected by long distances or peak traffic times, or even difficult situations, the time of performing CPR may be extended to dozens of minutes [32, 38]. In these dozens of minutes of on-site processing and transportation time, it is critical to perform and maintain high-quality CPR (with ideal compression quality, shortest "hand-off" time, and the best conditions for intervention) to achieve ROSC. According to statistics, less than $11 \%$ of patients with OHCA can survive to discharge $[39,40]$.

In the current situation in Taiwan, for example, non-urban areas are faced with uneven distribution of emergency-related equipment and personnel. In general, ambulances are equipped with one driver with one EMT. If an OHCA patient is transported, the EMT in the patient compartment can only perform CPR as much as possible before arriving at the hospital, and no one is available for turn-taking. Given the insufficiency in the number of EMTs, it is crucial to delay the fatigue caused by performing chest compressions and maintain the quality of CPR. This study has confirmed that an EMT can maintain a high level of CPR quality and reduce fatigue by using an intermittent chest compression method, and interruptions of 20 seconds were found to be superior to interruptions of 10 seconds.

\section{Limitations}

The limitations of this study must be considered. First, the participants were male firefighters with EMT licenses and were qualified to perform the intermittent chest compression tests. The results of the study may not apply to female EMT, or the general public without professional first aid training or hospital personnel with poor physical fitness. Second, the simulator used (Little Anne) has an excessively low impedance, so the results could be different if another Laerdal simulator had been used, such as the Resusci Anne QCPR. Furthermore, this study only investigated the effects and feasibility of intermittent chest compressions on the quality of CPR by using the Manikin and did not directly investigate the survival rates of OHCA patients. It is hoped that the effect of intermittent chest compressions on the survival rate can be further investigated in the future.

\section{Conclusions}

Given the above findings, chest compressions without interruption at the emergency site and during transportation may not allow high-quality CPR to be maintained for a long time. This study has confirmed that the upper body muscle mass of a firefighter with an EMT license is positively correlated with the quality of CPR, and also that the fatigue caused by long-term chest compressions can be reduced, and better CPR quality can be maintained, through intermittent chest compressions. We expect that this method can be applied to first aid training courses and first aid scenes and can become one of the methods of maintaining CPR quality in difficult situations.

\section{Abbreviations}

EMTs: emergency medical technicians; CPR: cardiopulmonary resuscitation; CCD: chest compression depth; CCF: chest compression fraction; CCRR: chest compression rebound rate; RPE: ratings of perceived exertion; CCR: chest compression rate; OHCA: out-of-hospital cardiac arrest; ROSC: return of 
spontaneous circulation; AHA: American Heart Association; EMT-2: Emergency Medical TechnicianIntermediate; ULMM: upper limb muscle mass; CMM: core muscle mass; UL+CMM: upper limb + core muscle mass; ANOVA: analysis of variance; PBF: body fat percentage.

\section{Acknowledgements}

The authors would like to thank all the participants of this study and their respective fire department for their assistance, and the members of the Sports Technology Laboratory for their assistance in the preparation and implementation of the study.

\section{Ethics Committee Approval and Patient Consent}

The study procedure was approved by the Institutional Review Board of $\mathrm{Fu}$ Jen Catholic University (New Taipei City, Taiwan) (reference number: C108165).

\section{Competing Interests}

The authors have declared that no competing interest exists.

\section{References}

1. Kalra A, Maharaj V, Johannsen RA, Hollenberg SM. Catheterization laboratory activation during mechanical cardiopulmonary resuscitation: when should we say "no?" Catheter Cardiovasc Interv. 2014; 83: 58-64.

2. Abella BS, Sandbo N, Vassilatos P, et al. Chest compression rates during cardiopulmonary resuscitation are suboptimal: a prospective study during in-hospital cardiac arrest. Circulation. 2005; 111: 428-34.

3. Nishisaki A, Nysaether J, Sutton R, et al. Effect of mattress deflection on CPR quality assessment for older children and adolescents. Resuscitation. 2009; 80: 540-5.

4. Wallace SK, Abella BS, Becker LB. Quantifying the effect of cardiopulmonary resuscitation quality on cardiac arrest outcome a systematic review and meta-analysis. Circ Cardiovasc Qual Outcomes. 2013; 6: 148-56.

5. Xanthos T, Karatzas T, Stroumpoulis K, et al. Continuous chest compressions improve survival and neurologic outcome in a swine model of prolonged ventricular fibrillation. Am J Emerg Med. 2012; 30: 1389-94.

6. Ko YC, Hsieh MJ, Ma MH, et al. The effect of system performance improvement on patients with cardiac arrest: A systematic review. Resuscitation. 2020; 157: 156-65.

7. Brooks SC, Bigham BL, Morrison LJ. Mechanical versus manual chest compressions for cardiac arrest. Cochrane Database Syst Rev. 2011; 19: CD007260.

8. Chi CH, Tsou JY, Su FC. Effects of rescuer position on the kinematics of cardiopulmonary resuscitation $(\mathrm{CPR})$ and the force of delivered compressions. Resuscitation, 2008; 76: 69-75.

9. Meaney PA, Bobrow BJ, Mancini ME, et al. Cardiopulmonary resuscitation quality: improving cardiac resuscitation outcomes both inside and outside the hospital: a consensus statement from the American Heart Association. Circulation. 2013; 128: 417-35.

10. Hasegawa T, Daikoku R, Saito S, Saito Y. Relationship between weight of rescuer and quality of chest compression during cardiopulmonary resuscitation. J Physiol Anthropol. 2014; 33: 16.

11. Zhang FL, Yan L, Huang SF, Bai XJ. Correlations between quality indexes of chest compression. World J Emerg Med. 2013; 4: 54-8.

12. Kleinman ME, Brennan EE, Goldberger ZD, et al. Part 5: adult basic life support and cardiopulmonary resuscitation quality: 2015 American Heart Association Guidelines Update for Cardiopulmonary Resuscitation and Emergency Cardiovascular Care. Circulation. 2015; 132: S414-35
13. Travers AH, Rea TD, Bobrow BJ, et al. Part 4: CPR overview: 2010 American Heart Association Guidelines for Cardiopulmonary Resuscitation and Emergency Cardiovascular Care. Circulation. 2010; 122: S676-84.

14. Atkins DL, Berger S, Duff JP, et al. Part 11: pediatric basic life support and cardiopulmonary resuscitation quality: 2015 American Heart Association Guidelines Update for Cardiopulmonary Resuscitation and Emergency Cardiovascular Care. Pediatrics. 2015; 132: S519-25.

15. Ashton A, McCluskey A, Gwinnutt CL, Keenan AM. Effect of rescuer fatigue on performance of continuous external chest compressions over 3 min. Resuscitation. 2002; 55: 151-5.

16. López-González A, Sánchez-López M, Garcia-Hermoso A, et al. Muscular fitness as a mediator of quality cardiopulmonary resuscitation. Am J Emerg Med. 2016; 34: 1845-9.

17. Russo SG, Neumann $P$, Reinhardt $S$, et al. Impact of physical fitness and biometric data on the quality of external chest compression: a randomised, crossover trial. BMC Emerg Med. 2011; 11: 20.

18. Kaminska $\mathrm{H}$, Wieczorek $\mathrm{W}$, Matusik $\mathrm{P}$, et al. Factors influencing high-quality chest compressions during cardiopulmonary resuscitation scenario, according to 2015 American Heart Association Guidelines. Kardiol Pol. 2018; 76: 642-7.

19. Yasuda Y, Kato Y, Sugimoto K, et al. Muscles used for chest compression under static and transportation conditions. Prehosp Emerg Care. 2013; 17: $162-9$

20. Bucki B, Waniczek D, Michnik R, et al. The assessment of the kinematics of the rescuer in continuous chest compression during a 10-min simulation of cardiopulmonary resuscitation. Eur J Med Res. 2019; 24 : 9.

21. Ock SM, Kim YM, Chung Jh, Kim SH. Influence of physical fitness on the performance of 5-minute continuous chest compression. Eur J Emerg Med. 2011; 18: 251-6.

22. Hightower $\mathrm{D}$, Thomas $\mathrm{SH}$, Stone $\mathrm{CK}$, et al Decay in quality of closed-chest compressions over time. Ann Emerg Med. 1995; 26: 300-3.

23. Barcala-Furelos R, Abelairas-Gomez C, Romo-Perez V, Palacios-Aguilar J. Effect of physical fatigue on the quality CPR: a water rescue study of lifeguards: physical fatigue and quality $\mathrm{CPR}$ in a water rescue. Am J Emerg Med. 2013; 31: 473-7.

24. National Fire Agency, MOI, Taiwan (2019). White paper on fire service. Available at https://ebook.nfa.gov.tw/whitepaper/108/\#p=1 (accessed on 6 November 2020).

25. Min MK, Yeom SR, Ryu JH, et al. A 10-s rest improves chest compression quality during hands-only cardiopulmonary resuscitation: a prospective, randomized crossover study using a manikin model. Resuscitation. 2013; 84: $1279-84$

26. Brouwer TF, Walker RG, Chapman FW, Koster RW. Association Between Chest Compression Interruptions and Clinical Outcomes of Ventricular Fibrillation Out-of-Hospital Cardiac Arrest. Circulation. 2015; 132: 1030-7.

27. Ong MEH, Perkins GD, Cariou A. Out-of-hospital cardiac arrest: prehospital management. Lancet. 2018; 391: 980-8.

28. Hoehn EF, Cabrera-Thurman MK, Oehler J, Vukovic A, et al. Enhancing CPR During Transition From Prehospital to Emergency Department: A QI Initiative. Pediatrics. 2020; 145: e20192908.

29. Halhalli HC, Şancı E, Uslu T. The Comparison of Manual and Mechanical Chest Compression on Survival and Long-Term Neurological Outcome of Nontraumatic Out-of-Hospital Cardiac Arrest Patients. J Emerg Med. 2020; 59: 680-6.

30. Miller RM, Chambers TL, Burns SP. Validating InBody ${ }^{\circledR} 570$ Multi-frequency Bioelectrical Impedance Analyzer versus DXA for Body Fat Percentage Analysis. Journal of Exercise Physiology. 2016; 19: 71-8.

31. Ogata H, Fujimaru I, Kondo T. Degree of exercise intensity during continuous chest compression in upper-body-trained individuals. J Physiol Anthropol. 2015; 34: 43.

32. Chonde M, Escajeda J, Elmer J, et al. Challenges in the development and implementation of a healthcare system based extracorporeal cardiopulmonary resuscitation (ECPR) program for the treatment of out of hospital cardiac arrest. Resuscitation. 2020; 148: 259-65.

33. Bekgöz B, San İ, Ergin M. Quality Comparison of the Manual Chest Compression and the Mechanical Chest Compression during difficult Transport Conditions. J Emerg Med. 2020; 58: 432-8.

34. Berg RA, Sanders AB, Kern KB, et al. Adverse hemodynamic effects of interrupting chest compressions for rescue breathing during cardiopulmonary resuscitation for ventricular fibrillation cardiac arrest. Circulation. 2001; 104: 2465-70.

35. Bobrow BJ, Clark LL, Ewy GA, et al. Minimally interrupted cardiac resuscitation by emergency medical services for out-of-hospital cardiac arrest. JAMA. 2008; 299: 1158-65.

36. Bobrow BJ, Spaite DW, Berg RA, et al. Chest compression-only CPR by lay rescuers and survival from out-ofhospital cardiac arrest. JAMA. 2010; 304: $1447-54$ 
37. Zou Y, Shi $\mathrm{W}$, Zhu $\mathrm{Y}$, et al. Rate at $120 /$ min provides qualified chest compression during cardiopulmonary resuscitation. Am J Emerg Med. 2015; 33: 535-8.

38. Chien $\mathrm{CY}$, Su YC, Lin CC, et al. Is 15 minutes an appropriate resuscitation duration before termination of a traumatic cardiac arrest? A case-control study. Am J Emerg Med. 2016; 34: 505-9.

39. Shinozaki K, Nonogi H, Nagao K, Becker LB. Strategies to improve cardiac arrest survival: a time to act. Acute Med Surg. 2016; 3: 61-4.

40. Libby C, Skinner RB, Rawal AR. EMS Termination Of Resuscitation And Pronouncement of Death. In: StatPearls [Internet]. Treasure Island (FL): StatPearls Publishing. 2020; PMID: 31082157. 